\title{
sciendo
}

\section{Application of Virtual Reality in Competitive Athletes - A Review}

\author{
by \\ Anna Akbaş ${ }^{1}$, Wojciech Marszałek ${ }^{1}$, Anna Kamieniarz ${ }^{1}$, Jacek Polechoński ${ }^{1}$, \\ Kajetan J. Stomka', Grzegorz Juras ${ }^{1}$
}

\begin{abstract}
The purpose of this study was to determine the state of the art in the area of virtual reality in competitive athletes of different levels of expertise in various disciplines and point the areas of its application. Articles published before August 2018 were considered in our review. The PubMed, SCOPUS, SportDiscus and Medline databases were searched. A combination of the following search terms was used: virtual reality, virtual environment, virtual system, athletes, sports, physical training, sport performance, physical exercises. Studies involved healthy competitive athletes. A total of 18 articles met the inclusion criteria. There were three areas of application of virtual reality to sport: performance analysis, simulation improvement and virtual training. Competitive athletes were mostly examined in a semi-immersive setting. In conclusion, virtual reality seems to play a marginal role in competitive athletes' training. Due to the fact that virtual reality interventions bring significant improvements in clinical research, well-designed randomized control trials with detailed virtual training programmes are required in the future. Practically, virtual reality is effectively and commonly used to analyse performance in competitive athletes. There is still a need of creating fully interactive VR, where athletes will be able to cooperate with a virtual partner and influence the environment.
\end{abstract}

Key words: sport simulation, immersion, training, athletic performance.

\section{Introduction}

One of the fundamental aims of participating in sport is to maximise athletes' performance. Research and innovation technology provide an increasing number of information about technical and tactical sport profiles, supporting physical training. In recent years, virtual reality technology (VR) has been often used in many areas of science, finding an application in military, education, mental health, medical therapy and sport.

VR is a visual-based computer simulation which can reproduce a realistic and controlled environment. Its definition is mostly determined by both perceiving and interacting within the created environment. VR is capable to generate modifications which cannot be done in a real world and hence, excites all the senses. In terms of neuroscience and behavioral health, VR training shows high potential in diagnostics and the treatment of mental health disorders. VR, as well as the real environment, is able to invoke embodied simulations of the human in the world, used to represent and predict actions, concepts, and emotions. VR can deceive the human brain's predictive coding mechanism and create a real feeling of being present in the virtual body and space (Riva et al., 2018).

The potential use of VR in athletes is based on its exceptional features. According to Düking et al. (2018) possibilities of modifications in presented scenarios and an infinite number of repetitions, which can be performed by athletes in unchanged conditions, are the greatest assets of this technology. VR also provides individualized training of technical and tactical as well as motor abilities regardless of the time and place, against a

1 - Institute of Sport Sciences, The Jerzy Kukuczka Academy of Physical Education, Katowice, Poland.

Authors submitted their contribution to the article to the editorial board.

Accepted for printing in the Journal of Human Kinetics vol. 69/2019 in September 2019. 
chosen opponent. Additionally, VR opens up possibilities in the context of research and diagnostics in sports, but also helps to practise motor imagery and managing physiological stress in competitive situations.

Application of interactive VR to sport-based tasks among healthy participants is dominated by cycling, running or rowing activity. In terms of sports performance, interaction means that physical effort influences virtual performance e.g. cycling speed (Neumann et al., 2017). VR is also used in training of sport-related skills in ball sports such as rugby, table tennis or soccer. In this case it is essential to provide informative or guidance feedback, which refers to the knowledge about the result or movement, respectively (Miles et al., 2012).

In this study we attempted to assess the use of VR in professional sports and athletes' training routines, excluding sport-like activities. Although previous studies are related to VR in sport, just a few of them involved experienced athletes or teams. As opposed to Miles et al. (2012), who reviewed VR training in ball sports, this study aim was to show the state of the art in the area of VR in competitive athletes at different levels of expertise in various sport disciplines and determine the spectrum of its application. Moreover, we provided detailed characteristics of examined athletes and VR systems, including the level of engagement and creation of the virtual environment (VE).

\section{Methods}

\section{Participants}

Only studies performed on competitive and healthy athletes were included. Athletes were not restricted by age, sex, discipline, fitness or competitive levels. A control group was not required. The characteristics of participants are summarized in Table 1.

\section{Design and Procedures}

The search of four databases (SPORTDiscus, PubMed, Medline and Scopus) was conducted using different combinations of the following terms: (virtual reality OR, virtual environment OR, virtual system) AND (athletes OR, sports OR, physical training OR, sport performance OR, physical exercises). Literature search was completed in August, 2018. Early screening of the articles based on titles and abstracts was performed by two authors (AK and WM). Full-text articles were independently assessed by other authors (AA and KS).

Publications search was not time-restricted. Studies were chosen under the following groups of inclusion criteria: 1) language (articles written in English language), 2) publication type (research studies or articles, conference proceedings), 3) publication status (only published articles with full-text available), 4) participants (at least one group of subjects were healthy, competitive athletes), 5) type of outcome measures (performance-based, self-reported), 6) articles were included even if there was no control group. Studies were excluded if: 1) the article covered leisure activity, recreation or physical education, 2) the main task was not specific for one sport discipline, 3) the aim of the study was post-injury rehabilitation or injury prevention, 4) the study type was a systematic review, meta-analysis or another non-empirical article, 5) there was no VR or the type of VR was not precise, and 6) the article focused only on virtual sports simulator construction and did not involve human subjects.

To avoid ambiguity, the selection of studies was precisely analysed. For example, Cortes et al. (2011) referred to anterior cruciate ligament injury risk factors, however, the aim of that study was to assess differences between unanticipated and anticipated lower extremity biomechanics while performing a sidestep cutting task. Direct implications resulting from research did not contain information about injury prevention or rehabilitation. Based on the above facts, we decided to include that study in our review.

\section{Measures}

Two reviewers (AA and WM) extracted the following information independently from the included studies: (1) characteristics of participants (including age, body mass and height, sport discipline, sports level and training experience), (2) type of VR used (including the level of immersion, type of display, supporting systems, and creation of the VE), (3) main findings. Data were collected and organized in Tables 1, 2 and 3. Due to the heterogeneity of variables, study populations, and study design used in studies to utilize VR in competitive athletes, systematic review and metaanalysis of data were not possible. 


\section{Results}

Computerized literature search included four databases and contained a total number of 1613 articles. After title selection and the removal of duplicates, abstracts of 399 original research papers were reviewed for eligibility. Full text of 77 articles was evaluated in detail. Finally, 18 articles met inclusion criteria. Figure 1 presents the flowchart for the literature search process.

The total sample size in reviewed articles was 385 subjects among who 233 were competitive athletes of different levels of expertise. The majority of athletes were experts, members of national teams or had international competition experience. Less often, athletes competed at collegiate or regional levels. Characteristics of participants divided into groups of individual and team sports are shown in Table 1.

Typically, expert groups were compared to novice. Nevertheless, Craig et al. (2006) compared prediction of the curved free kick trajectory in soccer in goalkeepers and field players showing non-significant effects of position on the pitch on the percentage of a given goal responses. Also, Tsai et al. (2017), in order to compare VR and tabletbased basketball training tactics used two groups of basketball players. Although the experimental group that used a VR simulator showed shorter average hesitation time in learning tactics in comparison to the tablet-based group, statistical difference between groups was not provided due to high intra-group variability.

There are three levels of engagement with VE: full, semi and non-immersive VR. The level of immersion is based on a set of technical manipulations and does not refer to the sense of presence. Full-immersion enhances users' individual experience and a subjective feeling of presence. That is why these two terms, immersion and presence, cannot be completely separated although the difference between them should be noticed. Included articles were assigned to the immersion levels according to the type of VR display used (Table 2).

Fully immersive VR utilizes headmounted displays (HMD) as well as a computer assisted virtual environment (CAVE) in evaluation of performance, e.g. in ball sports such as rugby (Brault et al., 2012), soccer (Craig et al., 2006; Dessing and Craig, 2010; Vignais et al., 2009) and basketball (Covaci et al., 2012; Tsai et al.,
2017). Petri et al. (2018) provides a fair insight into the preferable display mode during the development of an autonomous character executing attack against a real athlete in karate kumite. Athletes were asked whether a CAVE or an HMD would be more appropriate for their training. Using the Igropus Presence Questionnaire and Simulator Sickness Questionnaire the answers of expert karate athletes were grouped in 3 categories: good, moderate and poor, and scaled from -3 to 3 . The results plead in favour to the HMD $(1.875 \pm 0.25)$ over the CAVE display method $(0.25 \pm 0.5)$. According to Brault et al. (2012) immersive and interactive VR provides the most realistic training environment. Firstly, the CAVE and HMD assisted with a headtracking system, produce high quality visualizations which are observed from the first-person viewpoint and completely fill the user's field of view. Secondly, real tracking of moving athlete provides a direct link between VE perceptual information and undertaken action. In implementation of a basketball free-throw simulator, visual feedback was used as a simple form of interaction with a trainee (Covaci et al., 2012). In the situation of an unsuccessful shot, software provided immediate information about the variables of the shot, such as direction and speed of the ball, and instructed how to perform it more accurately in the next attempt (e.g. higher, lower). Finally, animated characters present often real captured movement (Brault et al., 2012; Petri et al., 2018) and actions, such as the ball trajectory which relays on theoretical aerodynamical models (Craig et al., 2009, 2006) what makes the VE even more realistic. The data are summarized in Table 2.

Commonly, semi-immersive VR engages a smaller field of view in comparison to immersive conditions and allows a trainee to look 'outside' the VE during the session. Scenery is projected on big, flat or cylindrical screens of different quantity and curvature. In some studies (Bideau et al., 2004; Brault et al., 2015; Tyreman et al., 2008; Vignais et al., 2009), the feeling of presence was enhanced by stereoscopic vision enabled just with special glasses. This solution allows for a larger degree of freedom than the HMD and consequently, allows to perform whole body actions, i.e. goalkeeping tasks (Bideau et al., 2004; Brault et al., 2015; Vignais et al., 2015). Tyreman et al. (2008) underwent exploration of differences in reaction time and 
anticipation time in a three and two-dimensional reality for ice hockey goaltenders. Both groups of subjects were slower in their anticipation time under three-dimensional conditions. The authors suggest that the part of the slowdown may be based on tracking information provided by the stereoscopic vision which could improve experts' and novices' ability to anticipate. These results are in accordance with Vignais et al. (2015). Anticipation abilities under stereoscopic conditions were also examined in baseball batters, where experts were significantly more accurate in predicting the type of the pitch in the uncoupled response mode (Ranganathan and Carlton, 2007).

A unique approach was used by Tornese et al. (2011) in order to reweight visuovestibular sensory inputs and indirectly improve balance performance in figure skaters. Athletes underwent video training sessions while standing on an unstable board, with the video projection on a 15.4inch laptop screen; the room was darkened in order to create a semi-immersive stimulation. Based on the previous definition, that type of immersion relies more on technical manipulations and does not refer to the sense of presence, thus, in contrary to Tornese et al. (2011), we decided to classify that study as non-immersive VR. Nonimmersive VR also refers to two or three dimensions viewed on an ordinary computer screen. A 17-inch notebook with 3D golf course visualization was also used to investigate expertise differences in the visual search behaviour of golfers of different performance levels in a simulated green-reading task (Campbell and Moran, 2014). Those researchers calculated duration and the number of fixations with the use of an eye-gaze binocular tracker.

The most popular data collection method in the analysed studies was a motion capture system. It was also common to judge final running direction of an opponent or an incoming ball direction using typical computer peripherals such as a gamepad or a computer mouse. Three studies used surveys or questionnaires as well as a video camera and different types of trackers following the changes in a position of the head, hand, eyes and even the baseball bat. Only few studies applied force plates, electromyography and electrooculography (Tab 3).

The professional athletes' opinion about VR training simulators was assessed in two studies
(Covaci et al., 2012; Petri et al., 2018). Although, based on these opinions, it is not possible to specify guidelines for the construction of a simulator, the provided data contain some interesting implications. First, Covaci et al. (2012) proposed that an element of rivalry could enhance the motivation and excitement during free-throws e.g. information about the opponent's score. Second, the realistic visualisation of computer-generated character and trainee's ability to respond adequately to character actions was the key factor in choosing the HMD over the CAVE in karate (Petri et al., 2018). Although the questionnaires used are not very precise, they seem to be reasonable tools in the assessment of VR simulators in athletes by indicating their weakest and strongest points. The usefulness of a windsurfing VR simulator for improving the pumping rate (i.e. moving a sail in order to use a wind) was also assessed. In contrast to previous studies, Ouadahi et al. (2016) empirically confirmed that the presence of VR during pumping improved athlete's performance in all of three measured variables: force, heart rate and cadence. Consequently, we can suppose that training in the VE may be a motivator itself.

There is a special place for VR dedicated to understanding goalkeeper's performance. Semiimmersive VR induced a significantly higher number of correct responses to the incoming ball, lower radial error and better height perception than video simulation (Vignais et al., 2015). These results support semi-immersive technology as a promising tool in analysing goalkeepers' actions. In ice hockey, saving a goal strategy is based on the shot type (backhand or forehand), however, the goalkeeper presents also the real time adjustment at the moment when the puck leaves the stick (Tyreman et al., 2008). Not just goalkeepers, but also advanced and novice field players take into account current ball heading direction and ball velocity in making a prediction where the ball will end (Craig et al., 2009; Dessing and Craig, 2010). In contrast, the human visual system cannot cope with recognition of lateral ball acceleration (Craig et al., 2006). Even expert goalkeepers are not able to fully compensate this visual difficulty and often make mistakes in the initial moment of a save. Experts dominate in coupled tasks, which consist of both anticipation and interception of the upcoming event (Dessing and Craig, 2010). The 
ball trajectory simulation was also expanded by suitable virtual kicker motion (Brault et al., 2015). No significant differences were found in time to react and radial error in goalkeeping performance when a thrower was animated at different graphical levels (from realistic animation to a humanoid created from markers attached to joints). However, different amounts of visual information significantly changed the goalkeeper's kinematics. The authors suppose that performance was regulated from different information sources (Vignais et al., 2009).

Both novice and expert athletes obtain information about the upcoming action from various kinematic variables. Experts are more sensitive to honest signals (e.g. centre of mass displacement) which reveal real opponent's body running direction. Novices are more likely to focus on deceptive movements (e.g. head or upper trunk deviation) (Brault et al., 2012). It appears that experts have an ability to accurately predict the result of an attacking action with less information. These outcomes are consistent with Vignais et al. (2009). Finally, Ranganathan and Carlton (2007) found direct evidence that baseball batters adjusted their movements relaying on the pitcher's kinematics. The initiation and duration of the batter's step occurred to be significantly related to the kind of the pitcher's throw- change-up or fastball. However, prediction accuracy of expert batters was primarily associated with visual information. Limited predictive skills can lead to different changes in movement kinematics, what exactly happened during unanticipated cutting manoeuvres in soccer (Cortes et al., 2011).

A totally different approach was used by Campbell and Moran (2014) in a decision-making study in the green-reading task among golfers of different expertise levels. Subjects were asked to make a prediction on where they would aim to try to successfully hole the prospective putt. The results based on the gaze pattern analysis showed that experts displayed a lower number of average visual fixations although of longer duration.

All the reviewed studies had a high risk of bias due to the lack of blinding, randomization, small sample size and diversified intensity, duration, and type of the experiment.

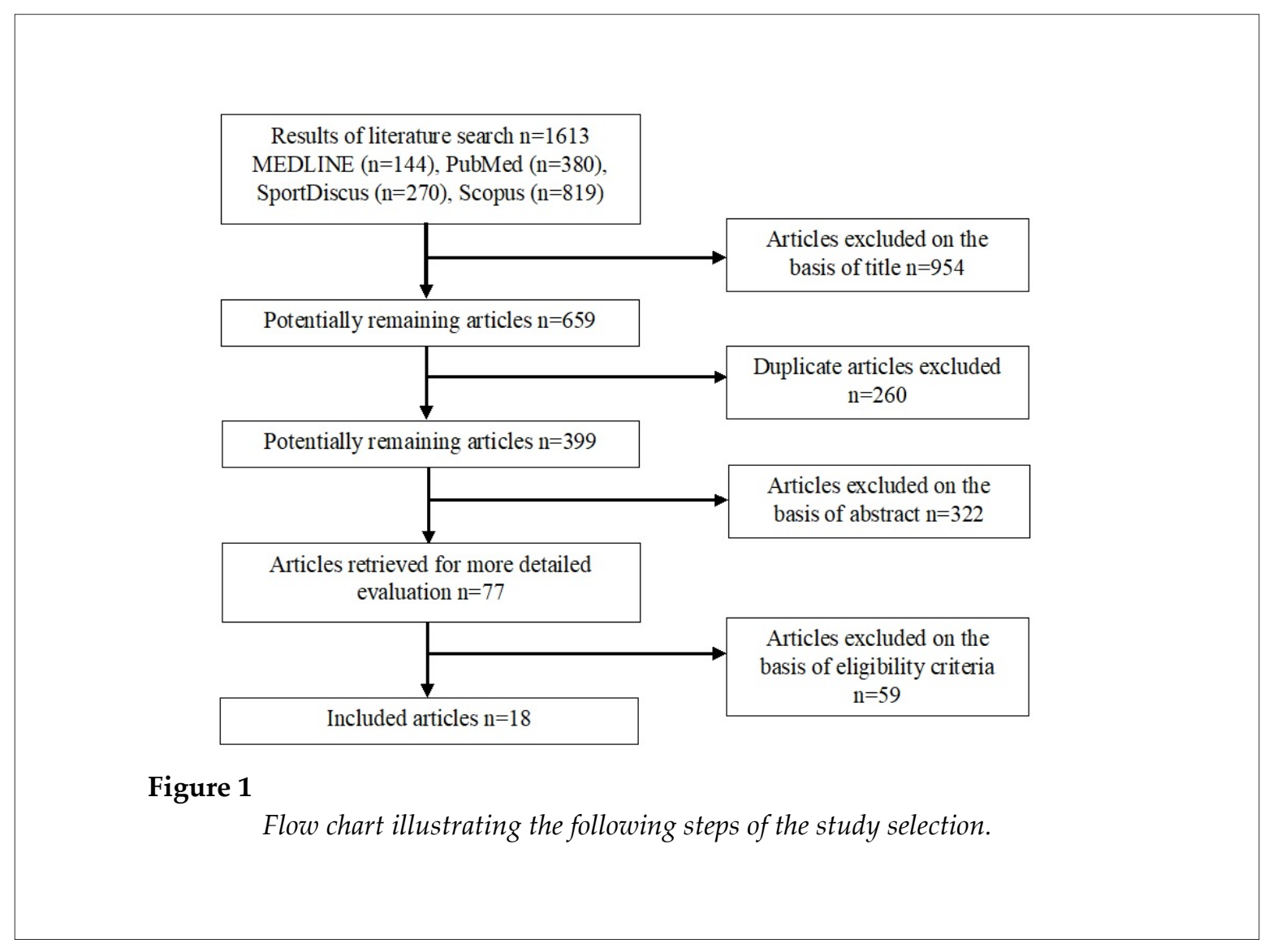




\section{Table 1}

Basic characteristic of subjects in investigated studies

\begin{tabular}{|c|c|c|c|c|c|c|c|c|c|c|}
\hline \multirow[b]{2}{*}{ Sport } & \multirow[b]{2}{*}{ Source } & \multirow[b]{2}{*}{ Gr } & \multirow[b]{2}{*}{ N subjects } & \multicolumn{2}{|c|}{ Gender } & \multicolumn{3}{|c|}{ Groups characteristics } & \multirow{2}{*}{$\begin{array}{l}\text { Level of } \\
\text { competition }\end{array}$} & \multirow{2}{*}{$\begin{array}{l}\text { Experience } \\
\text { [years] }\end{array}$} \\
\hline & & & & $\mathbf{F}$ & $\mathbf{M}$ & Age [years] & $\begin{array}{c}\text { Weight } \\
{[\mathrm{kg}]}\end{array}$ & $\begin{array}{l}\text { Height } \\
{[\mathrm{m}]}\end{array}$ & & \\
\hline \multirow{3}{*}{ golf } & \multirow{3}{*}{$\begin{array}{l}\text { Campbell } \\
\text { et al., } 2014\end{array}$} & E & 17 elite golfers & NR & NR & $24.4 \pm 2.91$ & NR & NR & $\begin{array}{l}\text { Irish Prof. } \\
\text { Golfers Assoc. }\end{array}$ & NR \\
\hline & & $\mathrm{C} 1$ & $\begin{array}{l}14 \text { elite } \\
\text { amateurs }\end{array}$ & NR & NR & $23.0 \pm 5.53$ & NR & NR & $\begin{array}{l}\text { Golfing Union } \\
\text { of Ireland }\end{array}$ & NR \\
\hline & & $\mathrm{C} 2$ & 14 amateurs & NR & NR & $30.78 \pm 14.34$ & NR & NR & local golf clubs & NR \\
\hline \multirow{2}{*}{$\begin{array}{c}\text { ice } \\
\text { skating }\end{array}$} & \multirow{2}{*}{$\begin{array}{l}\text { Tornese et } \\
\text { al., } 2011\end{array}$} & E & 5 figure skaters & 3 & 2 & 22.5 & NR & NR & $\begin{array}{c}\text { Italian National } \\
\text { Team }\end{array}$ & NR \\
\hline & & $\mathrm{C}$ & 23 novices & 13 & 10 & 22.7 & NR & NR & none & none \\
\hline karate & $\begin{array}{c}\text { Patri et al., } \\
2018\end{array}$ & $\mathrm{E}$ & 6 karatekas & NR & NR & NR & NR & NR & NR & NR \\
\hline \multirow{2}{*}{$\begin{array}{l}\text { windsu } \\
\text { rfing }\end{array}$} & ¿ Ouadahia & $E$ & 3 windsurfers & NR & NR & $14 \pm 1$ & $60 \pm 1$ & $1.70 \pm 0.1$ & NR & $2 \pm 1$ \\
\hline & et al., 2016 & $\mathrm{C}$ & 6 students & NR & NR & $23 \pm 4$ & $60 \pm 11$ & $1.70 \pm 0.1$ & none & none \\
\hline \multirow{4}{*}{ basebal } & Gray et & $E$ & 8 baseballers & 0 & 8 & $19-26$ & NR & NR & collegiate & $14.2 \pm 0.5$ \\
\hline & al., 2007 & $\mathrm{C}$ & 8 novices & 0 & 8 & $19-26$ & NR & NR & none & $5.1 \pm 0.6$ \\
\hline & 11 Ranganat & $\mathrm{E}$ & 10 baseballers & 0 & 10 & $20.2 \pm 1.0$ & NR & NR & university team & NR \\
\hline & $\begin{array}{c}\text { han et al., } \\
2007\end{array}$ & $\mathrm{C}$ & 10 novices & 0 & 10 & $23.3 \pm 3.6$ & NR & NR & $\begin{array}{c}\text { familiar with } \\
\text { the game }\end{array}$ & NR \\
\hline \multirow{3}{*}{$\begin{array}{c}\text { basket } \\
\text { ball }\end{array}$} & $\begin{array}{c}\text { Covaci, et } \\
\text { al., } 2012\end{array}$ & $\mathrm{E}$ & 5 basketballers & NR & NR & NR & NR & NR & NR & NR \\
\hline & Tsai et al., & $\mathrm{E}$ & 30 basketballers & 5 & 25 & $18-32$ & NR & NR & NR & $<3$ to $>10$ \\
\hline & 2017 & $\mathrm{C}$ & 30 basketballers & 14 & 16 & $18-32$ & NR & NR & NR & $<3$ to $>10$ \\
\hline \multirow{9}{*}{ soccer } & $\begin{array}{l}\text { Brault et } \\
\text { al., } 2015\end{array}$ & $\mathrm{E}$ & 11 goalkeepers & NR & NR & $26.1 \pm 4.1$ & NR & NR & regional & $16.4 \pm 5.4$ \\
\hline & $\begin{array}{c}\text { Cortese et } \\
\text { al., } 2011\end{array}$ & $\mathrm{E}$ & $\begin{array}{c}13 \text { soccer } \\
\text { players }\end{array}$ & 13 & 0 & $19.3 \pm 0.9$ & $61.3 \pm 5.6$ & $1.68 \pm 0.05$ & Division I & NR \\
\hline & & $\mathrm{E}$ & $\begin{array}{l}11 \text { elite soccer } \\
\text { players }\end{array}$ & NR & NR & 25.1 & NR & NR & $\begin{array}{l}\text { AC Milan, } \\
\text { Olympique de }\end{array}$ & NR \\
\hline & $\begin{array}{l}\text { Craig et } \\
\text { al., } 2006\end{array}$ & $\mathrm{C}$ & $\begin{array}{c}9 \text { elite } \\
\text { goalkeepers }\end{array}$ & NR & NR & 25.1 & NR & NR & $\begin{array}{l}\text { Marseille, } \\
\text { Bayer } \\
\text { Leverkusen, } \\
\text { Schalke } 04\end{array}$ & NR \\
\hline & \multirow{3}{*}{$\begin{array}{l}\text { Craig et } \\
\text { al., } 2009\end{array}$} & $\mathrm{E}$ & $\begin{array}{c}20 \text { elite soccer } \\
\text { players ( } 9 \\
\text { goalkeepers) }\end{array}$ & NR & NR & 25.1 & NR & NR & as above & NR \\
\hline & & $\mathrm{C} 1$ & 13 novices & 0 & 13 & $22-31$ & NR & NR & none & none \\
\hline & & $\mathrm{C} 2$ & 8 novices & 0 & 8 & $24-39$ & NR & NR & none & none \\
\hline & \multirow[t]{2}{*}{$\begin{array}{c}\text { Dessing et } \\
\text { al., } 2010\end{array}$} & $E$ & 2 goalkeepers & NR & NR & 16 and 30 & NR & NR & $\begin{array}{l}\text { U16, Irish } \\
\text { Premier League }\end{array}$ & 5 and 12 \\
\hline & & $\mathrm{C}$ & 10 novices & NR & NR & $29 \pm 4.7$ & NR & NR & none & none \\
\hline \multirow{3}{*}{$\begin{array}{c}\text { hand } \\
\text { ball }\end{array}$} & $\begin{array}{l}\text { Bideau et } \\
\text { al., } 2004\end{array}$ & $\mathrm{E}$ & 8 goalkeepers & NR & NR & NR & NR & NR & $\begin{array}{c}\text { National } \\
\text { French League }\end{array}$ & NR \\
\hline & $\begin{array}{l}\text { Vignais et } \\
\text { al., } 2009\end{array}$ & $E$ & 10 goalkeepers & 0 & 10 & $23.5 \pm 4.5$ & $85.3 \pm 12.2$ & $1.86 \pm 0.07$ & $\begin{array}{c}\text { French } \\
\text { Division I }\end{array}$ & NR \\
\hline & $\begin{array}{c}\text { Vignais et } \\
\text { al., } 2015\end{array}$ & E & 10 goalkeepers & 0 & 10 & $24 \pm 5.2$ & $85.9 \pm 14.4$ & $1.84 \pm 0.04$ & $\begin{array}{c}\text { French } \\
\text { Division I }\end{array}$ & NR \\
\hline \multirow{2}{*}{$\begin{array}{c}\text { ice } \\
\text { hokey }\end{array}$} & \multirow{2}{*}{$\begin{array}{l}\text { Tyreman } \\
\text { et al., } 2007\end{array}$} & E & 9 goaltenders & 3 & 6 & 28 & NR & NR & $\begin{array}{c}\text { university, } \\
\text { varsity teams }\end{array}$ & NR \\
\hline & & $\mathrm{C}$ & 10 novices & 4 & 6 & 27 & NR & NR & none & NR \\
\hline \multirow{4}{*}{ rugby } & \multirow{4}{*}{$\begin{array}{l}\text { Brault et } \\
\text { al., } 2012\end{array}$} & E1 & $\begin{array}{l}14 \text { rugby } \\
\text { players }\end{array}$ & NR & NR & $23.4 \pm 2.3$ & NR & NR & European & $13.3 \pm 5.6$ \\
\hline & & $\mathrm{C} 1$ & 14 novices & NR & NR & $22.6 \pm 3.3$ & NR & NR & none & none \\
\hline & & E2 & $\begin{array}{l}12 \text { rugby } \\
\text { players }\end{array}$ & NR & NR & $23.9 \pm 2.9$ & NR & NR & NR & NR \\
\hline & & $\mathrm{C} 2$ & 12 novices & NR & NR & $22.6 \pm 2.6$ & NR & NR & none & none \\
\hline
\end{tabular}


Table 2

Characteristics of virtual reality systems and virtual environment in investigated studies

\begin{tabular}{|c|c|c|c|c|c|}
\hline LI & Source & Display & Additional systems & Interaction & VE/ humanoid creation \\
\hline \multirow{7}{*}{ 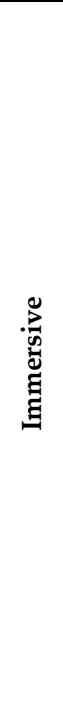 } & $\begin{array}{l}\text { Brault et } \\
\text { al., } 2012\end{array}$ & $\begin{array}{l}\text { HMD } \\
\text { (Cybermind } \\
\text { Visette) }\end{array}$ & $\begin{array}{c}\text { Intersense wireless (IS 900) } \\
\text { head tracker }\end{array}$ & $\begin{array}{l}\text { real-time head } \\
\text { interaction }\end{array}$ & $\begin{array}{l}\text { real-life 3D motion capture } \\
\text { recordings }\end{array}$ \\
\hline & $\begin{array}{l}\text { Covaci et } \\
\text { al. } 2012\end{array}$ & CAVE & $\begin{array}{l}\text { optical tracker } \mathrm{H} .27 \text {, real } \\
\text { basketball hoop }\end{array}$ & $\begin{array}{l}\text { visual } \\
\text { feedback }\end{array}$ & NR \\
\hline & $\begin{array}{l}\text { Craig et al., } \\
\quad 2006\end{array}$ & $\begin{array}{l}\text { HMD } \\
\text { (Cybermind Hi- } \\
\text { Res900) }\end{array}$ & $\begin{array}{l}\text { head tracking system } \\
\text { (Flock of Birds } \\
\text { electromagnetic sensor) }\end{array}$ & $\begin{array}{l}\text { real-time head } \\
\text { interaction }\end{array}$ & $\begin{array}{l}\text { theoretical model incorporating } \\
\text { aerodynamic Magnus-Robins lift } \\
\text { and drag forces }\end{array}$ \\
\hline & $\begin{array}{l}\text { Craig et al., } \\
2009\end{array}$ & $\begin{array}{l}\text { HMD } \\
\text { (Cybermind Hi- } \\
\text { Res900) }\end{array}$ & $\begin{array}{l}\text { head tracking system } \\
\quad \text { (Flock of Birds } \\
\text { electromagnetic sensor) }\end{array}$ & $\begin{array}{l}\text { real-time head } \\
\text { interaction, } \\
\text { performance } \\
\text { feedback }\end{array}$ & $\begin{array}{l}\text { validated aerodynamics model } \\
\text { including gravity, drag, and } \\
\text { Magnus - Robins lift forces }\end{array}$ \\
\hline & $\begin{array}{l}\text { Dessing et } \\
\text { al., } 2010\end{array}$ & $\begin{array}{l}\text { HMD } \\
\text { (Cybermind } \\
\text { Visette) }\end{array}$ & NR & $\begin{array}{c}\text { visual } \\
\text { feedback }\end{array}$ & $\begin{array}{l}\text { theoretical model incorporating } \\
\text { aerodynamic Magnus-Robins lift } \\
\text { and drag forces }\end{array}$ \\
\hline & $\begin{array}{l}\text { Petri et al., } \\
2018\end{array}$ & $\begin{array}{l}\text { CAVE, HMD } \\
\text { (Oculus) }\end{array}$ & NR & interactive & $\begin{array}{l}\text { infrared motion capturing } \\
\text { system VICON }\end{array}$ \\
\hline & $\begin{array}{c}\text { Tsai et al., } \\
2017\end{array}$ & $\begin{array}{l}\text { HMD (HTC } \\
\text { Vive) }\end{array}$ & $\begin{array}{c}\text { electronic 2D BTB } \\
\text { (Basketball Tactics Board) }\end{array}$ & $\begin{array}{l}\text { real-time head } \\
\text { interaction }\end{array}$ & NR \\
\hline \multirow{9}{*}{ 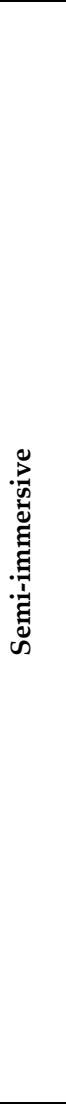 } & $\begin{array}{l}\text { Bideau et } \\
\text { al., } 2004\end{array}$ & $\begin{array}{l}\text { large cylindrical } \\
\text { screen }\end{array}$ & $\begin{array}{l}\text { stereovision glasses, } 3 \\
\text { synchronised video } \\
\text { projectors (Barco 1208S), } \\
\text { real goal }\end{array}$ & $\begin{array}{l}\text { real-time head } \\
\text { interaction }\end{array}$ & $\begin{array}{c}\text { throwers' motion } \\
\text { capture, resulting model is } \\
\text { based on statistically averaged } \\
\text { motions }\end{array}$ \\
\hline & $\begin{array}{l}\text { Brault et } \\
\text { al., } 2015\end{array}$ & $\begin{array}{l}\text { stereoscopic } \\
\text { projector (Acer } \\
\text { H5360) }\end{array}$ & $\begin{array}{l}\text { light glasses (Nvidia 3D), } \\
\text { real goal, synthetic grass } \\
\text { on the floor }\end{array}$ & $\begin{array}{l}\text { real-time head } \\
\text { interaction }\end{array}$ & $\begin{array}{l}\text { motion capture of attacker, } \\
\text { motion capture of the ball } \\
\text { (dynamical model parameters) }\end{array}$ \\
\hline & $\begin{array}{l}\text { Cortese et } \\
\text { al., } 2011\end{array}$ & screen & projector & $\begin{array}{l}\text { non } \\
\text { interactive }\end{array}$ & $\begin{array}{l}\text { ball trajectory changed using } \\
\text { given equations }\end{array}$ \\
\hline & $\begin{array}{l}\text { Gray et al., } \\
2007\end{array}$ & screen & $\begin{array}{l}\text { Gray's batting simulation, } \\
\text { projector, real bat }\end{array}$ & $\begin{array}{l}\text { audio } \\
\text { feedback }\end{array}$ & $\begin{array}{l}\text { motion toward the batter was } \\
\text { simulated by increasing the } \\
\text { ball's angular size }\end{array}$ \\
\hline & $\begin{array}{l}\text { Ouadahia } \\
\text { et al., } 2016\end{array}$ & screen & $\begin{array}{l}\text { fan system, audio system, } \\
\text { sailboard simulator }\end{array}$ & interactive & $\begin{array}{l}\text { high graphic quality video game } \\
\text { representing a sailboard on sea }\end{array}$ \\
\hline & $\begin{array}{l}\text { Ranganath } \\
\text { an et al., } \\
2007\end{array}$ & screen & $\begin{array}{c}\text { video projectors, } \\
\text { lightweight polarized } \\
\text { goggles }\end{array}$ & $\begin{array}{c}\text { non } \\
\text { interactive }\end{array}$ & $\begin{array}{l}\text { 10-camera motion-analysis } \\
\text { system to capture the kinematics } \\
\text { of the pitcher, } 2 \text { standard } \\
\text { cameras ball flight }\end{array}$ \\
\hline & $\begin{array}{l}\text { Tyreman et } \\
\text { al., } 2007\end{array}$ & screen & $\begin{array}{l}\text { stereoscoping glasses, } \\
\text { plastic ice hockey net, } \\
\text { hockey goaltender gloves, } \\
\text { full sized straight } \\
\text { bladedhockey goaltender, } \\
\text { Flock of Birds, video } \\
\text { projector }\end{array}$ & $\begin{array}{l}\text { non } \\
\text { interactive }\end{array}$ & twin-lens camera \\
\hline & $\begin{array}{l}\text { Vignais et } \\
\text { al., } 2009\end{array}$ & cylindrical screen & $\begin{array}{c}\text { stereoscopic glasses,real } \\
\text { goal }\end{array}$ & $\begin{array}{l}\text { real-time head } \\
\text { interaction }\end{array}$ & $\begin{array}{l}\text { VICON motion capture system } \\
\text { (throwing movements) }\end{array}$ \\
\hline & $\begin{array}{l}\text { Vignais et } \\
\text { al., } 2015\end{array}$ & screen & real goal & $\begin{array}{c}\text { non } \\
\text { interactive }\end{array}$ & $\begin{array}{l}\text { VICON MX40, } 12 \text { cameras, } 40 \\
\text { reflective markers, } 6 \text { on the ball }\end{array}$ \\
\hline \multirow{2}{*}{ 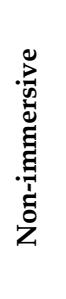 } & $\begin{array}{l}\text { Campbell } \\
\text { et al., } 2014\end{array}$ & notebook sc & NR & $\begin{array}{c}\text { non } \\
\text { interactive }\end{array}$ & $\begin{array}{l}\text { three-dimensional, computer- } \\
\text { generated golf green was } \\
\text { designed }\end{array}$ \\
\hline & $\begin{array}{c}\text { Tornese et } \\
\text { al., } 2011\end{array}$ & notebook screen & $\begin{array}{l}\text { motorized rotating chair, } \\
\text { unstable platform }(40 \times 40 \\
\mathrm{cm}) \text { with a cylindrical } \\
\text { curved base, digital } \\
\text { photocamera }\end{array}$ & $\begin{array}{c}\text { non } \\
\text { interactive }\end{array}$ & $\begin{array}{l}\text { images and sounds acquired in } \\
\text { MPEG-4 format by a digital } \\
\text { photocamera (1st person view) }\end{array}$ \\
\hline
\end{tabular}

LI- level of immersion, NR-non-reported 
Table 3

Summary of main findings of reviewed articles.

\begin{tabular}{|c|c|c|c|}
\hline Source & Task & Data aquisition & Main findings \\
\hline $\begin{array}{l}\text { Bideau et } \\
\text { al., } 2004\end{array}$ & $\begin{array}{l}\text { intercepting } \\
\text { randomly presented } \\
\text { throws }\end{array}$ & $\begin{array}{l}\text { motion capture } \\
\text { system } \\
\text { (Vicon370) }\end{array}$ & $\begin{array}{l}\text { Modified throws produced significant differences in the } \\
\text { goalkeepers' movements while identical throws caused } \\
\text { identical gestures. }\end{array}$ \\
\hline \multirow{2}{*}{$\begin{array}{l}\text { Brault et al., } \\
\quad 2012\end{array}$} & $\begin{array}{l}\text { judge final running } \\
\text { direction }\end{array}$ & gamepad & $\begin{array}{l}\text { Experts are attuned to honest signals that specify future } \\
\text { running direction; novices are more attuned to deceptive } \\
\text { signals. }\end{array}$ \\
\hline & $\begin{array}{l}\text { judge final running } \\
\text { direction, 'tackle' the } \\
\text { attacker }\end{array}$ & gamepad & $\begin{array}{l}\text { Experts were able to guide their actions to successfully } \\
\text { intercept the attacking player. }\end{array}$ \\
\hline $\begin{array}{l}\text { Brault et al., } \\
\quad 2015\end{array}$ & $\begin{array}{l}\text { intercept different } \\
\text { free kick conditions }\end{array}$ & $\begin{array}{l}\text { motion capture } \\
\text { system } \\
(\text { Vicon370) }\end{array}$ & $\begin{array}{l}\text { A wall of } 5 \text { players is the best choice a soccer goalkeeper } \\
\text { must have. }\end{array}$ \\
\hline $\begin{array}{l}\text { Campbell } \\
\text { and Moran, } \\
2014\end{array}$ & golf green-reading & $\begin{array}{l}\text { eye-gaze } \\
\text { binocular eye } \\
\text { tracker (LC } \\
\text { Technologies } \\
\text { 2005) }\end{array}$ & $\begin{array}{c}\text { Distinctive periods of visual perceptual- cognitive activity } \\
\text { may be evident in athletes' pre-performance routines-as } \\
\text { they "toured" the environment in which skill execution was } \\
\text { required. }\end{array}$ \\
\hline $\begin{array}{l}\text { Cortese et } \\
\text { al., } 2011\end{array}$ & $\begin{array}{l}\text { a sidestep cutting } \\
\text { task, running stop- } \\
\text { jump }\end{array}$ & $\begin{array}{l}\text { motion capture } \\
\text { system (Vicon), } \\
\text { Bertec Force } \\
\text { Plates (Model } \\
\text { 4010) }\end{array}$ & $\begin{array}{l}\text { Novel visualization software may (1) imitate the demands of } \\
\text { a practice/game situation in a laboratory environment; (2) use } \\
\text { a realistic scenario similar to what participants experience in } \\
\text { their daily routine when compared to light and arrows } \\
\text { stimulus. }\end{array}$ \\
\hline $\begin{array}{l}\text { Covaci et } \\
\text { al., } 2012\end{array}$ & shoot free throws & $\begin{array}{l}\text { survey, visual } \\
\text { observation }\end{array}$ & $\begin{array}{l}\text { Professional players obtained in the VE results similar to the } \\
\text { real environment; simulator can be used for beginners as an } \\
\text { interesting and stimulating tool to learn. }\end{array}$ \\
\hline $\begin{array}{l}\text { Craig et al., } \\
\quad 2006\end{array}$ & $\begin{array}{l}\text { judge if ball ended up } \\
\text { in the goal or not }\end{array}$ & computer mouse & $\begin{array}{l}\text { Visual system is not attuned to such accelerated motion, } \\
\text { which may explain why goalkeepers appear to misjudge the } \\
\text { future arrival point of such curved free kicks. }\end{array}$ \\
\hline $\begin{array}{l}\text { Craig et al., } \\
2009\end{array}$ & $\begin{array}{l}\text { judge whether the ball } \\
\text { would have entered } \\
\text { the goalmouth or not }\end{array}$ & comput & $\begin{array}{l}\text { An analysis of potential informational variables that could } \\
\text { explain these results points to the use of a first-order } \\
\text { compound variable combining optical expansion and optical } \\
\text { displacement. }\end{array}$ \\
\hline $\begin{array}{l}\text { Dessing et } \\
\text { al., } 2010\end{array}$ & saving a ball & $\begin{array}{c}\text { head tacker } \\
\text { (Intersense } \\
\text { motion IS-900), } \\
\text { hand tracker } \\
\text { (Microtrax } \\
\text { Intersense) }\end{array}$ & $\begin{array}{l}\text { Goalkeepers' movements were influenced by spin direction, } \\
\text { reflecting the limited sensitivity to visual acceleration of the } \\
\text { human visual system. Given the complexity of real-life free- } \\
\text { kick situations, goalkeepers cannot always fully compensate } \\
\text { for this limitation. }\end{array}$ \\
\hline $\begin{array}{l}\text { Gray et al., } \\
\quad 2007\end{array}$ & hitting the ball & $\begin{array}{l}\text { Fastrak } \\
\text { (Polhemus) } \\
\text { position tracker }\end{array}$ & $\begin{array}{l}\text { Empirical proof for intuition was not found, nor a theoretical } \\
\text { account of how predictions were made. }\end{array}$ \\
\hline $\begin{array}{l}\text { Ouadahia } \\
\text { et al., } 2016\end{array}$ & pumping exercise & $\begin{array}{l}\text { sailboard } \\
\text { simulator }\end{array}$ & $\begin{array}{l}\text { VR apparatus improved performance and acted like a } \\
\text { motivation enhancer. }\end{array}$ \\
\hline $\begin{array}{l}\text { Petri et al., } \\
2018\end{array}$ & $\begin{array}{l}\text { observation of the } \\
\text { autonomous character }\end{array}$ & $\begin{array}{l}\text { questionnaires } \\
\text { IPQ and SSQ }\end{array}$ & Karate athletes prefer a HMD over a CAVE. \\
\hline $\begin{array}{l}\text { Ranganatha } \\
\text { n et al., } \\
2007\end{array}$ & $\begin{array}{l}\text { verbal predictions to } \\
\text { pitcher, hitting the } \\
\text { virtual ball }\end{array}$ & $\begin{array}{l}\text { camera motion- } \\
\text { analysis system } \\
\text { (Motion } \\
\text { Analysis) }\end{array}$ & $\begin{array}{l}\text { The prediction accuracy of expert batters is primarily } \\
\text { associated with visual information of the ball rather than the } \\
\text { movement pattern of the pitcher. }\end{array}$ \\
\hline $\begin{array}{l}\text { Tornese et } \\
\text { al., } 2011\end{array}$ & $\begin{array}{l}\text { passive whole-body } \\
\text { rotations }\end{array}$ & $\begin{array}{l}\text { bitemporal DC- } \\
\quad \text { coupled } \\
\text { electrooculograp } \\
\text { hy (Toennies) }\end{array}$ & $\begin{array}{l}\text { The adopted VR technique was easy to perform in the usual } \\
\text { off-ice training context, and can be proposed as a low-cost } \\
\text { well-tolerated approach also for specific training of patients } \\
\text { with poor balance at least when oculomotor performance has } \\
\text { to be optimized. }\end{array}$ \\
\hline $\begin{array}{l}\text { Tsai et al., } \\
2017\end{array}$ & $\begin{array}{l}\text { executing a tactical } \\
\text { task }\end{array}$ & $\begin{array}{l}\text { camera, } \\
\text { questionnaire }\end{array}$ & $\begin{array}{l}\text { The user can practice in VR anytime even when there is no } \\
\text { coach, no sufficient trainees, or no empty basketball court. }\end{array}$ \\
\hline $\begin{array}{l}\text { Tyreman et } \\
\text { al., } 2007\end{array}$ & $\begin{array}{l}\text { goaltender reactions } \\
\text { to shots }\end{array}$ & video camera & $\begin{array}{l}\text { Goaltenders responses are based on the shot type and } \\
\text { reading the puck as it leaves the players stick, then plotting } \\
\text { an intercept. }\end{array}$ \\
\hline $\begin{array}{l}\text { Vignais et } \\
\text { al., } 2009\end{array}$ & saving the virtual shot & $\begin{array}{l}\text { motion capture } \\
\text { system (Vicon) }\end{array}$ & $\begin{array}{c}\text { Elite goalkeepers perform similarly for all graphical levels of } \\
\text { a virtual throwing action but kinematics differs for level } 3 \\
\text { and } 4 .\end{array}$ \\
\hline $\begin{array}{l}\text { Vignais et } \\
\text { al., } 2015\end{array}$ & saving $t$ & $\begin{array}{l}\text { motion capture } \\
\text { system (Vicon) }\end{array}$ & $\begin{array}{l}\text { Analysis of visual information uptake for handball } \\
\text { goalkeepers was better performed using a VR-based } \\
\text { methodology. }\end{array}$ \\
\hline
\end{tabular}




\section{Discussion}

The presented review synthesized information regarding the use of virtual reality in competitive athletes of different levels of expertise. Among the reviewed studies, we found three main research areas of interest. Mostly, VR was used to evaluate performance variables, especially in a goalkeeping task in handball, soccer and ice hockey. According to Bideau et al. (2010) VR is widely used in this field because of its unique opportunity to create standardized and replicable simulations in which only one variable can be evaluated at a time to determine its influence on athlete's performance. Producing complex manipulations in VR, such as the brightness of objects, their location, different perspective, temporal and spatial distortions of the movement trajectory, and feedback, may influence performance in ways that cannot be achieved in the real world. Secondly, athletes were often asked to evaluate effectiveness and utility of the VR simulator, or constituted the group of subjects who assisted in improving the system. Only in one study VR was used as a training tool to enhance athletes' motor abilities.

In the context of sports performance, the most frequently discussed topic was the anticipation of the upcoming action either if it was an approaching object or an opponent (Brault et al., 2012; Craig et al., 2009, 2006) or the result of the athlete's own action (Gray et al., 2007). In accordance with our assumptions, experts were significantly better in making predictions than novices (Brault et al., 2012; Ranganathan and Carlton, 2007; Tyreman et al., 2008). Experienced athletes were able to acquire information from the so called honest signals (i.e. centre of mass displacement) rather than from deceptive movements (Brault et al., 2012). Better technical skills and motor abilities allow them to observe an action for a longer time with precision (observe significant movement cues) and prepare more accurate adjustments (Ranganathan and Carlton, 2007; Tyreman et al., 2008). In contrast, there is one group of authors who found no significant differences between novices and experts in judging whether the ball would end up in or outside the goal (Craig et al., 2009, 2006). The brain dynamics associated with acquisition of the moving target in immersive VR shows that the visual processing of the target occurs before the onset of effector movements (i.e. eye, hand) and is accompanied by decreased amplitude of visual evoked potentials in brain activity over days of training (Clements et al., 2018).

Surprisingly, there is no application of VR in the area of specialized sports training among competitive athletes. Practising in a virtual environment has been widely used in novice athletes (Neumann et al., 2017), clinical trials and rehabilitation bringing tangible results (Juras et al., 2018). What is more, VR has become a common training tool in various high-skilled or high-risk occupational groups such as medical doctors, pilots and flight-crew or even in mining industry. Thus, we would like to stress that there is still a huge, unexploited potential in the area of VR training among athletes.

Only Tornese et al. (2011) introduced a VR training programme for figure skaters to improve their balance skills. Athletes underwent one training session for about 12-15 min a day for a total of 20 days involving the one-leg stance on an unstable platform with a cylindrically curved base. The proposed intervention brings significant modifications in vestibular adaptation in athletes and controls with reduced performance. The authors admit that the idea for such an intervention in figure skaters was taken from the VR treatment for people with vestibular disorders. There is evidence that plasticity of the neural system is 'use-dependent' and intensive and systematic practice may be necessary to modify its organization. Multisensory feedback potentiates the use-dependent plasticity process within the sensory-motor cortex, thus supporting motor learning or functional recovery (Calabrò et al., 2017). Using balance training programmes in different sport routines is very common. Nonetheless, the use of cheap, well-tolerated dryland drills in a non-interactive virtual environment appears as an innovative approach.

Virtual systems commonly consist of a large, often cylindrical, screen with stereoscopic glasses assisted by mechanical objects i.e. real goal. The biggest advantage of this setting for sport is ensuring a high level of freedom and creating a space where movements can be performed naturally. This type of configuration is considered semi-immersive VR. The second often used display is a fully immersive HMD. Although the cost of this technology is higher and the moving area is limited, HMDs provide vivid visualizations, allow 
for cognitive, psychomotor and affective skill acquisition. Affective skills refer to emotional responses to stressful or difficult situations (Rose et al., 2018).

It appears that realistic animation of a virtual opponent plays a key role in a semi-immersive setting (Bideau et al., 2004) and compensates the lack of full immersion. However, expert goalkeepers performed similarly irrespective of a graphical level of a virtual thrower. Only reducing the ball size slightly changed kinematics of arm saving action (Vignais et al., 2009). Noteworthy is that semi-immersive VR often benefits from additional equipment, for example, artificial grass, a real baseball bat or a fan system which stimulates additional cutaneous senses. To investigate the effects of a windsurfing digital simulation on user behaviour, motivation and physical performance Ouadahi et al. (2016) designed a sailboard simulator equipped with a fan and an audio system to simulate wind and water sounds. They observed an increase in average force of $20 \%$ for 8 subjects; furthermore, heart rate and the pumping frequency increased for all participants by an average value of 10 to $20 \%$ under VR conditions. Consequently, the developed apparatus connected to a VR windsurfing game improved the user performance and acted like a motivation enhancer. Regarding neurophysiology, this effect is associated with decreased perception of exertion during VR training. Moreover, studies have shown that critical variables in learning and relearning motor skills and in changing neural architecture are the quantity, duration and intensity of training sessions. Authors also suggest that the presence of avatar in virtual training may sustain the usedependent plastic changes within the higher sensory motor areas belonging to the mirror neuron system (Adamovich et al., 2009; Calabrò et al., 2017).

In the reviewed studies only three papers included full characteristics of the study group and almost every second study did not provide direct information about participants' gender. No comparison between male and female athletes was reported.

The greatest limitation of this review is a high diversity of included studies in the context of the study design, protocols used and outcome measures. Due to heterogeneity of the data nor the systematic juxtapositions of the result and procedures, neither meta-analysis could be performed.

Findings from this review are twofold. On the one hand, VR seems to play a surprisingly marginal role in competitive athletes' training, and consequently its effectiveness in sports training cannot be assessed. Due to the fact that VR interventions bring significant improvements in clinical research, well-designed randomized control trials with detailed VR training programmes are required in the future. On the other hand, VR is effectively and commonly used to analyse sports performance in competitive athletes. Performance evaluation in VR is primarily recommended to open-skill sports disciplines, where there is a need for constant adaptation to the changing behaviour of the opponents, and the conditions are completely unique. The coaches of team and combat sports (e.g. soccer, fencing, boxing, judo) would benefit most from repetitive VR conditions in the sense of understanding athlete's perception-action performance. There is still a need of creating fully interactive VR, where athletes will be able to cooperate with a virtual partner and influence the environment.

\section{References}

Adamovich SV, Fluet GG, Tunik E, Merians AS. Sensorimotor training in virtual reality: a review. NeuroRehabilitation, 2009; 25: 29-44

Bideau B, Kulpa R, Vignais N, Brault S, Multon F, Craig C. Using virtual reality to analyze sports performance. IEEE Comput Graph Appl, 2010; 30: 14-21

Bideau B, Multon F, Kulpa R, Fradet L, Arnaldi B, Delamarche P. Using virtual reality to analyze links between handball thrower kinematics and goalkeeper's reactions. Neurosci Lett, 2004; 372: 119-122

Brault S, Bideau B, Kulpa R, Craig CM. Detecting deception in movement: the case of the side-step in rugby. Plos One, 2012; 7

Brault S, Kulpa R, Duliscouët L, Marin A, Bideau B. Virtual kicker vs. real goalkeeper in soccer: a way to explore goalkeeper's performance. Mov Sport Sci - Sci Mot, 2015; 3: 79-88 
Calabrò RS, Naro A, Russo M, Leo A, De Luca R, Balletta T, Buda A, La Rosa G, Bramanti A, Bramanti P. The role of virtual reality in improving motor performance as revealed by EEG: a randomized clinical trial. $J$ Neuroeng Rehabil, 2017; 14: 53

Campbell MJ, Moran AP. There is more to green reading than meets the eye! Exploring the gaze behaviours of expert golfers on a virtual golf putting task. Cogn Process, 2014; 15: 363-372

Clements JM, Kopper R, Zielinski DJ, Rao H, Sommer MA, Kirsch E, Mainsah BO, Collins LM, Appelbaum LG. Neurophysiology of visual-motor learning during a simulated marksmanship task in immersive virtual reality. In: 25th IEEE Conference on Virtual Reality and 3D User Interfaces (VR). IEEE, 2018; 451458

Cortes N, Blount E, Ringleb S, Onate JA. Soccer-specific video simulation for improving movement assessment. Sport Biomech, 2011; 10: 22-34

Covaci A, Postelnicu CC, Panfir AN, Talaba D. A virtual reality simulator for basketball free-throw skills development. IFIP Adv Inf Commun Technol, 2012; 372: 105-112

Craig CM, Berton E, Rao G, Fernandez L, Bootsma RJ. Judging where a ball will go: The case of curved free kicks in football. Naturwissenschaften, 2006; 93: 97-101

Craig CM, Goulon C, Eric B, Rao G, Laure F, Reinoud JB. Optic variables used to judge future ball arrival position in expert and novice soccer players. Atten Percept Psychophys, 2009; 71: 481-489

Dessing JC, Craig CM. Bending it like Beckham: How to visually fool the goalkeeper. Plos One, 2010; 5

Düking P, Holmberg HC, Sperlich B. The potential usefulness of virtual reality systems for athletes: A short SWOT analysis. Front Physiol, 2018; 9: 1-4

Gray R, Beilock SL, Carr TH. "As soon as the bat met the ball, i knew it was gone": Outcome prediction, hindsight bias, and the representation and control of action in expert and novice baseball players. Psychon Bull Rev, 2007; 14: 669-675

Juras G, Brachman A, Michalska J, Kamieniarz A, Pawłowski M, Hadamus A, Białoszewski D, Błaszczyk J, Słomka KJ. Standards of virtual reality application in balance training programs in clinical practice: a systematic review. Games Health J, 2018; 8

Miles HC, Pop SR, Watt SJ, Lawrence GP, John NW. A review of virtual environments for training in ball sports. Comput Graph, 2012; 36: 714-726

Neumann DL, Moffitt RL, Thomas PR, Loveday K, Watling DP, Lombard CL, Antonova S, Tremeer MA. A systematic review of the application of interactive virtual reality to sport. Virtual Real, 2017

Ouadahi N, Chadli S, Ababou A, Ababou N. A simulator dedicated to strengthening exercises for windsurfers. Procedia Eng, 2016; 147: 532-537

Petri K, Witte K, Bandow N, Emmermacher P, Masik S, Dannenberg M, Salb S, Zhang L, Brunnett G. Development of an autonomous character in karate kumite. Advances in Intelligent Systems and Computing, 2018; 663

Ranganathan R, Carlton LG. Perception-action coupling and anticipatory performance in baseball batting. $J$ Mot Behav, 2007; 39: 369-380

Riva G, Wiederhold BK, Mantovani F. Neuroscience of virtual reality: from virtual exposure to embodied medicine. Cyberpsychology, Behav Soc Netw, 2018; 10

Rose T, Nam CS, Chen KB. Immersion of virtual reality for rehabilitation - review. Appl Ergon, 2018; 69: 153161

Tornese D, Botta M, Mattei V, Alpini DC. Self-experienced virtual reality to improve balance reflexes in ice dancers. A pilot study. Sport Sci Health, 2011; 6: 45-50

Tsai W-L, Chung M-F, Pan T-Y, Hu M-C. Train in virtual court: basketball tactic training via virtual reality. Proc 2017 ACM Work Multimedia-based Educ Knowl Technol Pers Soc Online Train, 2017; 3-10

Tyreman H, Parker JR, Katz L. Ice hockey goaltenders' strategies, reaction times and anticipation times in twoand three-dimensional virtual environments. In: IACSS, Preolympic Congress on Computer Science in Sport, 2008; 68-72

Vignais N, Bideau B, Craig C, Brault S, Multon F, Delamarche P, Kulpa R. Does the level of graphical detail of a virtual handball thrower influence a goalkeeper's motor response? J Sport Sci Med, 2009; 8: 501-508 
Vignais N, Kulpa R, Brault S, Presse D, Bideau B. Which technology to investigate visual perception in sport: Video vs. virtual reality. Hum Mov Sci, 2015; 39: 12-26

\title{
Corresponding author:
}

\author{
Anna Akbaş \\ Institute of Sport Sciences \\ The Jerzy Kukuczka Academy of Physical Education, \\ 72a Mikolowska Str., 40-065 Katowice, Poland \\ Phone/fax (+48) 322075174 \\ E-mail: a.akbas@awf.katowice.pl
}

